\title{
Editorial
}

Nephrology

Published online: March 13, 2018

\section{Addressing Racial Disparity in the Progression of Chronic Kidney Disease: Prescribe More Fruits and Vegetables?}

\author{
Wei Chen ${ }^{a}$ David A. Bushinsky ${ }^{a, b}$ \\ ${ }^{a}$ Department of Medicine, Rochester, NY, USA; ${ }^{b}$ Department of Pharmacology and Physiology, University of \\ Rochester School of Medicine and Dentistry, Rochester, NY, USA
}

Compared to ancestral human diets rich in plants which are metabolized to bases, contemporary diets displace many of these foods with animal proteins metabolized to acids. Adaptation to these dietary changes necessitates a marked increase in renal net acid excretion to maintain systemic acid-base balance. As kidneys fail, the ability to excrete acid diminishes and consumption of dietary acids leads to acid retention and metabolic acidosis [1]. Metabolic acidosis, generally defined by a reduced serum bicarbonate concentration, since blood $\mathrm{pH}$ is rarely measured in clinical studies, predicts chronic kidney disease (CKD) progression [2]. Several studies have shown that the correction of metabolic acidosis with sodium bicarbonate slows CKD progression [3]. The mechanism by which metabolic acidosis leads to a more rapid renal decline is unclear, but may involve increased single nephron ammoniagenesis, generation of aldosterone, and/or endothelin 1 [1].

Dietary acid load (DAL) is associated with CKD progression although this relationship is not as robust as that

\section{KARGER}

(c) 2018 S. Karger AG, Basel

E-Mail karger@karger.com

www.karger.com/ajn between low serum bicarbonate and CKD progression. In African Americans with hypertensive CKD, a higher DAL was associated with a faster decline in renal function, but time-to-event analyses showed that there was no association with a composite of renal events, defined as end stage renal disease (ESRD) or doubling of serum creatinine from baseline [4]. Among patients with CKD stages 2-4 from the Chronic Renal Insufficiency Cohort, a higher DAL was associated with a greater risk of CKD progression only among those without diabetes [5]. Interventional studies demonstrate that the addition of base-producing fruits and vegetables were comparable to sodium bicarbonate supplementation not only in correcting metabolic acidosis but in preserving renal function [3]. In a study of patients with hypertensive CKD stage 3 and a plasma bicarbonate level between 22 and $24 \mathrm{mEq} / \mathrm{L}$, participants eating base-producing fruits and vegetables had a slower decline in renal function and decreased urine $\mathrm{N}$-acetyl- $\beta$-D-glucosaminidase and angiotensinogen compared to usual care, and results were comparable 
to those given sodium bicarbonate [3]. The investigators made every effort to ensure compliance by providing free fruits and vegetables sufficient for the entire family in the intervention group; however, it is impossible to implement blinding in this type of dietary study.

In this issue of the American Journal of Nephrology, Crews et al. [6] examined whether there was a racial disparity in the relationship between DAL and the risk of ESRD. This group has previously reported the association between DAL and the risk of ESRD among 1,486 adults with CKD in the National Health and Nutrition Examination Survey (NHANES) III, which is a cohort of United States non-institutionalized civilians, conducted between 1988 and 1994 [7]. Participants with the highest tertile of DAL had a higher risk of progressing to ESRD over a median follow-up of 14 years compared to those with the lowest tertile (adjusted relative hazard: 3.04 [95\% CI 1.58-5.86]). The current study examined the same relationship in NHANES III but focused the analyses on nonHispanic blacks (NHB) and non-Hispanic whites (NHW; $n=1,123)$ and found that race modified the relationship between DAL and the risk of ESRD ( $p$ for interaction = 0.004 ). Over a median of 7.5 year follow-up, NHB had a higher risk of ESRD with increasing DAL compared to NHW (adjusted hazard ratio [HR] 1.21 [95\% CI 1.121.31 ] vs. 1.08 [ $95 \%$ CI $0.96-1.20$ ] per $1 \mathrm{mEq} /$ day increase in DAL).

An important observational study, such as this one, is hypothesis generating. Race may modify the relationship between DAL and the risk of ESRD due to a genetic difference between NHB and NHW [8-10]. Hypertensive blacks are more likely to have a greater increase in blood pressure in response to dietary sodium than whites, perhaps due to an upregulated intrarenal renin-angiotensinaldosterone system in blacks [8]. DAL may result in metabolic acidosis, especially in patients with CKD, leading to further stimulation of aldosterone secretion which has adverse hemodynamic effects and induces renal fibrosis [10]. African Americans are also likely to have a high risk apolipoprotein $\mathrm{L} 1$ alleles which are associated with CKD progression [9] and these alleles may modify the relationship between DAL and CKD progression.

Inherent study biases and residual confounding could also be responsible for the observed difference between NHB and NHW. Study biases might result from dietary recall as a method to estimate DAL and handling of the missing data. In this study, NHB had a median DAL of $50.9 \mathrm{mEq} /$ day (interquartile range [IQR] 40.466.8), while in the African American Study of Kidney Disease and Hypertension trial patients with a similar glomerular filtration rate had a higher median DAL of $72.8 \mathrm{mEq} /$ day (IQR 57.2-89.5) [4]. The apparent difference in DAL between the 2 studies may result from the different methods used to estimate DAL. In the current study, DAL was estimated using 24-h dietary recall and calculated using the Remer and Manz net acid excretion formula while in the African American Study of Kidney Disease and Hypertension trial, DAL was estimated using net endogenous acid production and calculated from 24-h urine urea nitrogen and potassium excretion. While both methods of estimating DAL have drawbacks, dietary recall is limited by the subject's ability to accurately remember the diet that was consumed (recall bias) and the ability of the interviewer to impartially record a response (interviewer bias). These biases may result in differential misclassification, especially since the dietary habits and culture may differ among NHW, $\mathrm{NHB}$, and the interviewers. In NHANES III, a significant number of participants $(86 \%)$ had missing dietary data and were excluded from analyses. Whether the characteristics of these participants differed from those that responded to the dietary questionnaire is not known. Unless the lack of response to the questionnaire was random, the missing data could introduce non-response bias and influence the conclusions drawn from the analyses.

Despite adjusting for a comprehensive set of covariates in the Cox regression models, there could still be residual confounding. Compared to NHW, NHB had a higher risk of ESRD (adjusted HR 1.68 [95\% CI 1.18-2.38]). NHB are more likely to have earlier onset and poorer control of diabetes and hypertension compared to NHW [8]. This difference along with other factors such as choice of antihypertensive medications, the presence of proteinuria, dietary sodium intake, socioeconomic status, and access to healthcare [8] may all contribute to the racial disparity in CKD progression. Several of these factors are associated with DAL and may confound the relationship between DAL and the risk of ESRD. In this study, analyses were adjusted for many covariates including the presence of diabetes, hypertension, proteinuria and poverty. However, other covariates such as the severity, control, and duration of diabetes and hypertension (e.g., hemoglobin A $1 \mathrm{c}$ and blood pressure readings), and the choice of antihypertensive medications, especially the use of renin-angiotensin-aldosterone blockers, were not adjusted for and may alter the association between DAL and the risk of ESRD. In this study, great effort was made to adjust for confounders; however, one can never include all possible covariates. 
As Dr. Martin Luther King, Jr. said at the Medical Committee for Human Rights in 1966, "Of all the forms of inequality, injustice in health is the most shocking and inhumane". This study investigated a potential reason for the higher risk of ESRD in NHB compared to NHW and led to the hypothesis that eliminating the difference in DAL between NHB and NHW may attenuate the racial disparity in the risk of ESRD. Prior studies support that a reduction in DAL with fruits and vegetables will help preserve the renal function [3]. Unlike genetic factors, which we currently cannot modify, a reduction in DAL can be achieved through expanding access to dietary education and increasing the availability and affordability of baseproducing fruits and vegetables. As physicians, we often do not have the luxury of waiting for a definitive study before making recommendations to patients and policy makers. Promoting consumption of more fruits and veg- etables to decrease DAL, especially in NHB, appears to have little risk and significant potential for the reward of preserving renal function.

\section{Acknowledgments}

W.C. was supported by the American Society of Nephrology Carl W. Gottschalk Research Grant. D.A.B. was supported by the National Institutes of Diabetes and Digestive and Kidney Diseases (R01 DK075462). Both W.C. and D.A.B. were supported by the Renal Research Institute.

\section{Disclosure Statement}

W.C. declares no competing interests. D.A.B. is a consultant for Relyspa, Amgen, Sanofi/Genzyme, Vifor, and Tricida and has an equity interest in Amgen and Tricida.

\section{References}

1 Nagami GT, Hamm LL: Regulation of acidbase balance in chronic kidney disease. Adv Chronic Kidney Dis 2017;24:274-279.

2 Chen W, Abramowitz MK: Epidemiology of acid-base derangements in CKD. Adv Chronic Kidney Dis 2017;24:280-288.

3 Goraya N, Simoni J, Jo CH, Wesson DE: Treatment of metabolic acidosis in patients with stage 3 chronic kidney disease with fruits and vegetables or oral bicarbonate reduces urine angiotensinogen and preserves glomerular filtration rate. Kidney Int 2014;86:10311038.
4 Scialla JJ, et al: Net endogenous acid production is associated with a faster decline in GFR in African Americans. Kidney Int 2012;82:106-112.

5 Scialla JJ, et al: Higher net acid excretion is associated with a lower risk of kidney disease progression in patients with diabetes. Kidney Int 2017;91:204-215.

6 Crews DC, Banerjee T, Wesson DE, Morgenstern H, Saran R, Burrows NR, Williams DE, Powe NR: Race/ethnicity, dietary acid load, and risk of end-stage renal disease among US adults with chronic kidney disease. Am J Nephrol 2018;47:174-181.
7 Banerjee T, et al: High dietary acid load predicts ESRD among adults with CKD. J Am Soc Nephrol 2015;26:1693-1700.

8 Martins D, Agodoa L, Norris KC: Hypertensive chronic kidney disease in African Americans: strategies for improving care. Cleve Clin J Med 2012;79:726-734.

9 Parsa A, et al: APOL1 risk variants, race, and progression of chronic kidney disease. $\mathrm{N}$ Engl J Med 2013;369:2183-2196.

10 Ponda MP, Hostetter TH: Aldosterone antagonism in chronic kidney disease. Clin J Am Soc Nephrol 2006;1:668-677. 